\title{
AN INEQUALITY OF GRÜSS' TYPE FOR RIEMANN-STIELTJES INTEGRAL AND APPLICATIONS FOR SPECIAL MEANS
}

\author{
S. S. DRAGOMIR AND I. FEDOTOV
}

\begin{abstract}
In this paper we derive a new inequality of Grüss' type for Riemann-Stieltjes integral and apply it for special means (logarithmic mean, identric mean, etc...).
\end{abstract}

\section{Introduction}

In 1935, G. Grüss proved the following inequality which establishes a connection betwen the integral of the product of two fucntions and the product of the integrals:

$$
\left|\frac{1}{b-a} \int_{a}^{b} f(x) g(x) d x-\frac{1}{b-a} \int_{a}^{b} f(x) d x \cdot \frac{1}{b-a} \int_{a}^{b} g(x) d x\right| \leq \frac{1}{4}(\Phi-\phi)(\Gamma-\gamma)
$$

provided that $f$ and $g$ two integrable functions on $[a, b]$ and satisfying the condition

$$
\phi \leq f(x) \leq \Phi \text { and } \gamma \leq g(x) \leq \Gamma \text { for all } x \in[a, b] .
$$

The constant $\frac{1}{4}$ is the best possible one and is achieved for $f(x)=g(x)=\operatorname{sgn}\left(x-\frac{a+b}{2}\right)$.

For other similar results, generalizations for positive linear functionals, discrete versions, determinantal versions etc. see the Chapter X of the book [1] due to Mitrinović, Pečarić and Fink where further references are given.

In this paper we point out a Grüss' type inequality for Riemann-Stieltjes integral and apply it for special means, i.e., logarithmic mean, identric mean, etc..

\section{The Results}

The following result of Grüss' type holds:

Theorem 2.1. Let $f, u:[a, b] \rightarrow R$ be so that $u$ is L-lipschitzian on $[a, b]$, i.e.,

$$
|u(x)-u(y)| \leq L|x-y| \text { for all } x, y \in[a, b]
$$

Received January 14, 1998.

1991 Mathematics Subject Classification. 26D15, 26Dxx.

Key words and phrases. Grüss' inequality, Riemann-Stieltjes integral. 
$f$ is Riemann integrable on $[a, b]$ and there exists the real numbers, $m, M$ so that

$$
m \leq f(x) \leq M \text { for all } x \in[a, b] .
$$

Then we have the inequality

$$
\left|\int_{a}^{b} f(x) d u(x)-\frac{u(b)-u(a)}{b-a} \int_{a}^{b} f(t) d t\right| \leq \frac{L}{2}(M-m)(b-a),
$$

and the constsnt $\frac{1}{2}$ is sharp, in the sense that it can not be replaced by a smaller one.

Proof. First of all let observe that if $v$ is an $L$-lipschitzian mapping and $q$ is Riemann integrable on $[a, b]$, then

$$
\left|\int_{a}^{b} q(x) d v(x)\right| \leq L \int_{a}^{b}|q(t)| d t
$$

Indeed, if $\Delta_{n}: a=x_{0}^{n}<x_{1}^{n}<\cdots<x_{n-1}^{n}<x_{n}^{n}=b$ is a sequence of partitions of $[a, b]$ with $\nu\left(\Delta_{n}\right):=\max _{i=1, n-1}\left(x_{i+1}^{n}-x_{i}^{n}\right) \rightarrow 0$ (for $n \rightarrow \infty$ ) and $\xi_{i}^{n} \in\left[x_{i}^{n}, x_{i+1}^{n}\right]$ then

$$
\begin{aligned}
\left|\int_{a}^{b} q(x) d v(x)\right| & =\left|\lim _{\nu\left(\Delta_{n}\right) \rightarrow 0} \sum_{i=0}^{n-1} q\left(\xi_{i}^{n}\right)\left(v\left(x_{i+1}^{n}\right)-v\left(x_{i}^{n}\right)\right)\right| \\
& \leq \lim _{\nu\left(\Delta_{n}\right) \rightarrow 0} \sum_{i=0}^{n-1}\left|q\left(\xi_{i}^{n}\right)\right|\left|\frac{\left(v\left(x_{i+1}^{n}\right)-v\left(x_{i}^{n}\right)\right)}{x_{i+1}^{n}-x_{i}^{n}}\right|\left(x_{i+1}^{n}-x_{i}^{n}\right) \\
& \leq L \lim _{\nu\left(\Delta_{n}\right) \rightarrow 0} \sum_{i=0}^{n-1}\left|q\left(\xi_{i}^{n}\right)\right|\left(x_{i+1}^{n}-x_{i}^{n}\right)=L \int_{a}^{b}|q(t)| d t .
\end{aligned}
$$

Now, let observe that

$$
\begin{aligned}
& \left|\int_{a}^{b} f(x) d u(x)-\frac{u(b)-u(a)}{b-a} \int_{a}^{b} f(t) d t\right| \\
= & \left|\int_{a}^{b}\left(f(x)-\frac{1}{b-a} \int_{a}^{b} f(t) d t\right) d u(x)\right| \\
\leq & L \int_{a}^{b}\left|f(x)-\frac{1}{b-a} \int_{a}^{b} f(t) d t\right| d x .
\end{aligned}
$$

Now, define

Then we have

$$
I:=\frac{1}{b-a} \int_{a}^{b}\left(f(x)-\frac{1}{b-a} \int_{a}^{b} f(t) d t\right)^{2} d x
$$

$$
\begin{aligned}
I & =\frac{1}{b-a} \int_{a}^{b}\left[f^{2}(x)-2 f(x) \frac{1}{b-a} \int_{a}^{b} f(t) d t+\left(\frac{1}{b-a} \int_{a}^{b} f(t) d t\right)^{2}\right] d x \\
& =\frac{1}{b-a} \int_{a}^{b} f^{2}(x) d x-\left(\frac{1}{b-a} \int_{a}^{b} f(t) d t\right)^{2}
\end{aligned}
$$


and

$$
I=\left(M-\frac{1}{b-a} \int_{a}^{b} f(t) d t\right)\left(\frac{1}{b-a} \int_{a}^{b} f(t) d t-m\right)-\frac{1}{b-a} \int_{a}^{b}(M-f(t))(f(t)-m) d t
$$

As $m \leq f(x) \leq M$ for all $x \in[a, b]$, then

$$
\int_{a}^{b}(M-f(t))(f(t)-m) d t \geq 0
$$

which implies

$$
I \leq\left(M-\frac{1}{b-a} \int_{a}^{b} f(t) d t\right)\left(\frac{1}{b-a} \int_{a}^{b} f(t) d t-m\right) .
$$

Using the elemetary inequality

$$
(M-k)(k-m) \leq \frac{1}{4}[(M-k)+(k-m)]^{2}=\frac{1}{4}(M-m)^{2}
$$

which holds for $k, m, M \in R$, we get

$$
I \leq \frac{1}{4}(M-m)^{2}
$$

Using Cauchy-Buniakowski-Schwarz's integral inequality we have

$$
I \geq\left[\frac{1}{b-a} \int_{a}^{b}\left|f(x)-\frac{1}{b-a} \int_{a}^{b} f(t) d t\right| d x\right]^{2}
$$

Now, by (2.6), we get

$$
\int_{a}^{b}\left|f(x)-\frac{I}{b-a} \int_{a}^{b} f(t) d t\right| d x \leq \frac{1}{2}(M-m)(b-a)
$$

and then by (2.5) we obtain the desired inequality (2.3). To prove the sharpness of the constsnt $\frac{1}{2}$, let choose

$$
u(x):=\left|x-\frac{a+b}{2}\right|, f(x):=\operatorname{sgn}\left(x-\frac{a+b}{2}\right), x \in[a, b] .
$$

Then

$$
|u(x)-u(y)|=|| x-\frac{a+b}{2}|-| y-\frac{a+b}{2} \| \leq|x-y| \text { and all } x, y \in[a, b]
$$

which shows that $u$ is $L$-lipschitzian with the constsnt $L=1$. Also, because $-1 \leq f(x) \leq$ 1 , for all $x \in[a, b]$, then $M-m=2$ and

$$
\frac{L}{2}(M-m)(b-a)=b-a .
$$


On the other hand,

$$
\begin{aligned}
& \int_{a}^{b} f(x) d u(x)-\frac{u(b)-u(a)}{b-a} \int_{a}^{b} f(t) d t=\int_{a}^{b} \operatorname{sgn}\left(x-\frac{a+b}{2}\right) d u(x) \\
= & -\int_{a}^{\frac{a+b}{2}} d u(x)+\int_{\frac{a+b}{2}}^{b} d u(x)=-u\left(\frac{a+b}{2}\right)+u(a)+u(b)-u\left(\frac{a+b}{2}\right)=b-a
\end{aligned}
$$

which shows that the euqality is realised in (2.3).

Corollary 2.2. Let $f:[a, b] \rightarrow R$ be as above and $u:[a, b] \rightarrow R$ a differentiable mapping whose derivative $u^{\prime}:(a, b) \rightarrow R$ is bounded on $(a, b)$. Denote $\left\|u^{\prime}\right\|_{\infty}:=$ $\sup _{t \in(a, b)}\left|u^{\prime}(t)\right|<\infty$. Then we have the inequality

$$
\left|\int_{a}^{b} f(t) u^{\prime}(t) d t-\frac{u(b)-u(a)}{b-a} \int_{a}^{b} f(t) d t\right| \leq \frac{\left\|u^{\prime}\right\|_{\infty}}{2}(M-m)(b-a) .
$$

Corollary 2.3. Let $f:[a, b] \rightarrow R$ be a differentiable mapping whose derivative $f^{\prime}$ : $(a, b) \rightarrow R$ is bounded on $(a, b)$ and $f(a) \neq f(b)$. Denote $\left\|f^{\prime}\right\|_{\infty}:=\sup _{t \in(a, b)}\left|f^{\prime}(t)\right|<\infty$. Then we have the inequality

$$
\left|\frac{f(a)+f(b)}{2}-\frac{1}{b-a} \int_{a}^{b} f(t) d t\right| \leq \frac{\left\|f^{\prime}\right\|_{\infty}}{2|f(b)-f(a)|}(M-m)(b-a) .
$$

The proof is obvious from the above corollary choosing $u=f$.

Remark 2.4. If Corollary 2.2 we put $u=f^{p}, u=\ln f, u=\sin f$ etc $\cdots$, we can obtain some other intersting inequalities. We shall omit the details.

\section{Applications for Special Means}

We first discuss the application of the results in the previous sections to lower and upper bounds estimation of some important relationships between the following means:

The arithmetic mean: $A=A(a, b):=(a+b) / 2, \quad a, b \geq 0$.

The geometric mean: $G=G(a, b):=\sqrt{a b}, \quad a, b \geq 0$.

The harmonic mean:

$$
H=H(a, b):=\frac{2}{\frac{1}{a}+\frac{1}{b}}, \quad a, b>0 .
$$

The logarithmic mean:

$$
L=L(a, b):=\left\{\begin{array}{ll}
\frac{b-a}{\ln b-\ln a} & \text { if } a \neq b, \\
a & \text { if } a=b
\end{array}, \quad a, b>0\right.
$$


Note that for the convex mapping $f=\frac{1}{t}:(0, \infty) \mapsto \mathbb{R}$, we have

$$
\frac{1}{b-a} \int_{a}^{b} f(t) d t=L^{-1}(a, b) \text { for } a \neq b
$$

The $p$-logarithmic mean

$$
L_{p}=L_{p}(a, b):=\left\{\begin{array}{ll}
{\left[\frac{a^{p+1}-a^{p+1}}{(p+1)(b-a)}\right]^{1 / p}} & \text { if } a \neq b \\
a & \text { if } a=b
\end{array}, p \in \mathbb{R} \backslash\{-1,0\}, a, b>0 .\right.
$$

For the convex (or concave) mapping $f(t)=t^{p}, p \in(-\infty, 0) \cup[1, \infty) \backslash\{-1\}$ (or $\left.p \in(0,1)\right)$ we have

$$
\frac{1}{b-a} \int_{a}^{b} f(t) d t=L_{p}^{p}(a, b) \text { for } a \neq b
$$

The identric mean:

$$
I=I(a, b):=\left\{\begin{array}{ll}
\frac{1}{e}\left(\frac{b^{b}}{a^{a}}\right)^{\frac{1}{b-a}} & \text { if } a \neq b \\
a & \text { if } a=b
\end{array}, \quad a, b>0\right.
$$

For the convex mapping $f(t)=-\ln x$ we have

$$
\frac{1}{b-a} \int_{a}^{b} f(t) d t=\ln I(a, b) \text { if } a \neq b .
$$

These means are often used in numerical approximation and in other areas. However, the following simple relationships are known in the literature

$$
H \leq G \leq L \leq I \leq A
$$

It is also known that $L_{p}$ is monotonically increasing in $p \in \mathbb{R}$ with $L_{0}=I$ and $L_{-1}=L$.

We now derive various sophisticated bounds for some differences and products of the above special means using the results obtained in the previous section. These bounds are very useful in applications since the special means are often used in numerical approximations.

1. If in Corollary 2.2 we choose $f(x)=x^{q}, u(x)=x^{p+1}(p, q>0)$, then we get

$$
\left|L_{p+q}^{p+q}-L_{p}^{p} L_{q}^{q}\right| \leq \frac{q b^{p}}{2}(b-a) L_{q-1}^{q-1}
$$

2. If in Corollary 2.2 we choose $f(x)=x^{q}(q>0), u(x)=\frac{1}{x}$, and $a>0$, then we get

$$
\left|L_{q}^{q}-G^{2} L_{q-2}^{q-2}\right| \leq \frac{q}{2 a^{2}}(b-a) G^{2} L_{q-1}^{q-1} .
$$


3. If in the above corollary we choose $f(x)=x^{q}(q>0), u(x)=\ln x$, and $a>0$, then we get

$$
\left|L_{q}^{q}-L L_{q-1}^{q-1}\right| \leq \frac{q}{2 a^{2}}(b-a) L L_{q-1}^{q-1} .
$$

We remark that if in the above corollary we choose $f$ and $u$ in other appropriate ways, we get some other interesting inequalities for special means.

We omit the details.

\section{References}

[1] D. S. Mitrinović J. E. Pečarić and A. M. Fink, Classical and New Inequalities in Analysis, Kluwer Academic Publishers, Dordrecht, 1993.

Department of Mathematics, Victoria University of Technology, P.O. Box 14428, Melbourne City, MC, Victoria, Australia.

Department of Applied Mathematics, University of Transkei, Private Bag X1, UNITRA, UMTATA, 5100, South Africa. 